\title{
ANÁLISIS Y VALORACIÓN DE LOS SISTEMAS DE EVALUACIÓN DE SUELOS EN ESPAÑA. EVOLUCIÓN, TENDENCIAS ACTUALES Y PERSPECTIVAS FUTURAS
}

\author{
POR \\ C. AÑÓ VIDAL, J. SÁNCHEZ DÍAZ \\ y C. ANTOLÍN TOMÁS
}

\section{Introducción}

El objetivo fundamental de la evaluación de suelos es la selección, en cada una de las unidades cartográficas establecidas en el territorio, de la utilización más adecuada y menos agresiva con el entorno medioambiental, de acuerdo con los rasgos específicos que caracterizan los recursos biofísicos de un espacio territorial determinado. La consecución de este fin varía en función de los planteamientos metodológicos iniciales. Así, el proceso evaluador culmina con la evaluación de la idoneidad o aptitud de la tierra para acoger ciertos usos o sistemas de gestión específicos, o bien, con la evaluación de la capacidad de uso del suelo de una amplia gama de utilizaciones del medio de carácter general, aunque en ocasiones pueda integrar, implícitamente, un listado de prioridades. A pesar de plantear objetivos teóricamente bien definidos, los procedimientos metodológicos son muy variables $\mathrm{y}$, a veces, contradictorios, generando una elevada dosis de confusión, consecuencia, en muchas ocasiones, de interpretaciones deficientes de conceptos fundamentales o de la utilización im-

C. Añó Vidal, J. Sánchez Díaz y C. Antolín Tomás. U.D. Edafología. Departamento Biología Vegetal. Facultad de Farmacia. Universidad de Valencia.

Estudios Geográficos

Tomo LVII, n. ${ }^{\circ} 228$, julio-septiembre 1997 
precisa o incorrecta tanto de la nomenclatura como de los criterios que rigen los distintos sistemas de evaluación.

Esta caracerística, unido a la juventud de la disciplina, han determinado que la Evaluación de Suelos en España, dentro del contexto de la Edafología Aplicada, carezca, en estos momentos, de un marco metodológico bien estructurado que pudiera ser aceptado por el conjunto de la comunidad científica de la Ciencia del Suelo. En general, el panorama actual de la Evaluación de Suelos en nuestro país se caracteriza por utilizar variantes metodológicas de las contribuciones internacionales más significativas, adaptadas a condiciones ambientales muy concretas, o por la aplicación directa de los criterios de evaluación de las metodologías más aceptadas mundialmente. Esta peculiaridad de la disciplina es la principal consecuencia de un desarrollo tardío y una evolución muy dependiente de los sistemas de evaluación internacionales más relevantes, provocando que actualmente, aunque parezca sorprendente, no exista un marco metodológico propio, adaptado a las características biofísicas de nuestro territorio.

Los sistemas de evaluación más utilizados en España para evaluar el potencial de utilización de un suelo son los siguientes: la Clasificación de Capacidades Agrológicas, el método paramétrico de Riquier, Bramao y Cornet, y los procedimientos de evaluación establecidos por la Organización de las Naciones Unidas para la Agricultura y la Alimentación (F.A.O.).

Los tres métodos tienen un objetivo idéntico: establecer el uso más adecuado de los suelos, utilizando, sobre todo, la información generada por los reconocimientos edafológicos plasmada en los mapas de suelos. En estos documentos cartográficos el suelo está descrito, por regla general, en términos exclusivamente científicos que, en función de la terminología empleada, no aportan ninguna indicación sobre las posibilidades de utilización del recurso edáfico. Los procedimientos de evaluación interpretarán aquellas propiedades o características necesarias para poder evaluar la capacidad de utilización del suelo. Sin embargo, los criterios de evaluación establecidos en estas metodologías no son homogéneos, provocando, por un lado, que a partir de la misma fuente de información (la cartografía edafológica) las lecturas sean diferentes y, por otro, que las conclusiones de la evaluación no sean idénticas, aunque el objetivo sea similar.

A continuación se desarrollan los conceptos básicos y las venta- 
jas e inconvenientes que conllevan la aplicación de las metodologías más aceptadas en el ámbito científico de la cartografía y evaluación de suelos, síntesis del exhaustivo análisis que durantes los últimos años hemos realizado de los sistemas de evaluación tanto nacionales como internacionales (Añó, 1996), aportando un tipo de información muy difícil de encontrar en las metodologías de evaluación de suelos publicadas hasta el momento en España.

\section{Sistemas de Evaluación de Suelos en España. Características}

\section{Clasificación de Capacidades Agrológicas}

La Clasificación de Capacidades Agrológicas ha sido, durante muchos años, la metodología que más ha influido en los trabajos de evaluación desarrollados en España. Este sistema categórico, desarrollado en los Estados Unidos desde la década de 1930 y estructurado definitivamente por Klingebiel y Montgomery (1961), presenta tres características fundamentales:

1. El sistema, utilizando tres niveles de clasificación -clases, subclases y unidades- (Cuadro I), establece la capacidad de uso de la tierra en función de sistemas agronómicos descritos en términos muy generales y poco precisos, sin considerar las prácticas de manejo de cultivos específicos y sin valorar la introducción de mejoras importantes en el suelo (v. gr. regadío, drenaje, mejora de suelos afectados por salinidad, etc.). Con este fin agrupa las tierras en un número reducido de categorías jerarquizadas, diferenciando cada clase en función de las limitaciones que las propiedades permanentes del suelo, las características topográficas o las condiciones climáticas imponen a la utilización agrícola del medio que, además, implícitamente, es el uso potencialmente deseable.

2. La metodología concede una especial importancia a evitar o mitigar la erosión del suelo. El origen de este sistema de evaluación está íntimamente relacionado con los motivos que provocaron la constitución, en Estados Unidos, del Servicio de Conservación de Suelos: acelerada e intensa erosión del recurso edáfico, especiamente en el Medio Oeste, y la necesidad imperiosa de establecer tipos de 
CUADRO I

ESTRUCTURA DE LA CLASIFICACIÓN

DE CAPACIDADES AGROLÓGICAS (KLINGEBIEL y MoNTGOMERY, 1961)

\section{Clases Agrológicas}

Las clases de capacidad indican el sistema óptimo de explotación del suelo compatible con el mantenimiento de su capacidad productiva. En función de las limitaciones al uso agrícola que imponen las condiciones climáticas, topográficas y/o edáficas se establecen ocho clases denominadas con un número romano, del I al VIII. Las unidades con mayor capacidad son aquéllas que aceptan una amplia gama de usos del suelo sin riesgos de degradación.

\section{Subclases}

Identifica, añadiendo un subíndice a la clase, el tipo de limitación que aparece en cada unidad cartográfica. Se establecen cuatro tipos de limitaciones: riesgo de erosión (e), drenaje deficiente o riesgo de inundación $(w)$, limitaciones del suelo que afectan al desarrollo radicular $(s)$ y limitaciones de origen climático $(c)$.

\section{Unidades de Capacidad}

Agrupa unidades cartográficas con unas perspectivas de utilización uniformes, unas prácticas de conservación y gestión similares y una productividad potencial comparable. Se representan con la adición de un numeral árabe a la subclase. En muchas ocasiones, aunque no es necesario, las unidades de capacidad coinciden con la serie edáfica.

utilizaciones de la tierra que frenaron este tipo de degradación (Davidson, 1985).

3. El sistema parte de una premisa básica: los mapas edafológicos aportan el soporte imprescindible para poder establecer la capacidad de uso de los suelos. Únicamente, a partir de la interpretación de esta información básica y valorando las condiciones climáticas y topográficas puede establecerse la potencialidad del suelo, las limitaciones permanentes de su uso, el tipo de utilización más conveniente sin riesgos de erosión o las prácticas de conservación más adecuadas (Beek, 1981). De este modo, se transfiere al usuario de la evaluación la interpretación práctica de la información depositada en los mapas de suelos (Davidson, 1992).

Ventajas de la Clasificación de Capacidades Agrológicas.-La utilización de los planteamientos metodológicos de este sistema categórico 
de evaluación de suelos presenta muchas ventajas respecto a otros procedimientos, justificando la vigencia actual de esta metodología. Entre los aspectos positivos destacaríamos los siguientes:

1. Es un sistema de evaluación práctico y sencillo de aplicar. Además, la cartografía que refleja los resultados de la evaluación presenta un nivel de complejidad muy bajo.

2. Es un sistema muy flexible, rasgo común a todos los métodos de evaluación más difundidos y utilizados internacionalmente. Esta característica permite adaptar el sistema a las condiciones medioambientales específicas del área de estudio sin modificar la estructura básica de la clasificación.

3. La división del medio en un número reducido de categorías jerarquizadas facilita la comprensión de los resultados finales de la evaluación.

4. Las conclusiones de la evaluación, por regla general, concuerdan con las características físicas del área de estudio, evitando valoraciones erróneas que pueden producirse utilizando las fórmulas de los sistemas paramétricos (McRae y Burnham, 1981).

5. Esta valoración cualitativa del potencial del suelo y de sus limitaciones de uso aporta una visión general muy adecuada sobre la capacidad agrícola de los suelos, permitiendo diferenciar, rápidamente, entre las tierras susceptibles de ser cultivadas respecto a aquéllas que no lo pueden ser.

6. Al establecer el destino más apropiado de los terrenos de cultivo es un instrumento valioso par la planificación de los usos agrícolas del suelo (Dumanski et al., 1979).

Aplicación en España. Variantes metodológicas.-En España, la Dirección General de Producción Agraria del Ministerio de Agricultura (M.A.P.A., 1974), en un intento de crear un método oficial español, asimiló íntegramente los criterios de clasificación y evaluación del sistema desarrollado en Estados Unidos. Sin embargo, a diferencia de otros países que adaptaron el método original a sus condiciones medioambientales o a sus características socioeconómicas, el Ministerio de Agricultura introduce muy pocas modificaciones: considera un nuevo factor limitante (el contenido en carbonatos) y subdivide una clase, la IV, en tres subcategorías, 
diferenciadas en función de la intensidad de las prácticas de conservación que deben implantarse.

Existe una diferencia importante que es muy poco positiva: en Estados Unidos la evaluación se basa en estudios edafológicos muy detallados, publicados por regla general a escala 1:20.000 (Olson, 1984). Por el contrario en nuestro país la evaluación se realizará sin el soporte del mapa de suelos, salvo excepciones muy puntuales (Delgado y Aguilar, 1985; Badía et al., 1993). Por tanto, es muy discutible la exactitud y la fiabilidad de los resultados catográficos finales. Además, en un gran número de ocasiones el factor pendiente adquiere una relevancia excesiva ya que el establecimento de los diferentes intervalos es sencillo mediante los mapas topográficos (Porta et al., 1994).

En las mismas fechas, 1974, el Instituto de Edafología y Biología Vegetal del C.S.I.C. de Madrid, aplicó, en el estudio de los suelos del Area Metropolitana de Madrid, la adaptación portuguesa de la clasificación americana (Azevedo y Cardoso, 1962). El sistema portugués introduce pocas novedades: reduce el número de clases, de ocho a cinco, y modifica su denominación, de números romanos a letras, manteniendo los mismos niveles de clasificación y los principios metodológicos más imortantes (Cuadro II). Sin embargo, a diferencia de las aplicaciones efectuadas por el Ministerio de Agricultura, la evaluación se realizará a partir de estudios edafológicos previos que identifican y caracterizan los tipos de suelos y su distribución en el territorio objeto de estudio.

Una adaptación posterior es la metodología desarrollada en Valencia por Sánchez et al. (1984), que actuamente constituye, entre todos los sistemas categóricos de evaluación de suelos elaborados en España, la aportación metodológica mejor diseñada en función de las características ambientales mediterráneas. Este método introduce novedades muy interesantes, sobre todo la ampliación del número de factores limitantes y su cuantificación, y ha sido utilizado como instrumento de valoración del recurso suelo en la configuración del Sistema de Información Territorial de la Comunidad Valenciana (C.O.P.U.T., 1991). Además, es el sistema de evaluación que más ha influido en el desarrollo posterior de la metodologías de carácter categórico: v.gr. la elaboración de la Cartografía de la Capacidad Potencial de Uso Agrícola de la Comunidad de Madrid (Monturiol y Alcalá, 
ANÁLISIS Y VALORACIÓN DE LOS SISTEMAS DE EVALUACIÓN DE SUELOS...

CuAdro II

COMPARACIÓN APROXIMADA ENTRE EL SISTEMA DE CAPACIDADES AGROLÓGICAS (KLINGEBIEL Y MONTGOMERY, 1961) Y LA ADAPTACIÓN PORTUGUESA (Azevedo y Cardoso, 1962)

\begin{tabular}{|c|c|c|}
\hline \multicolumn{2}{|r|}{$\begin{array}{l}\text { Clases Clasificación Azevedo \& Cardoso. Definición } \\
\text { y características principales }\end{array}$} & \multirow{2}{*}{$\begin{array}{l}\begin{array}{l}\text { Clases Clasificación } \\
\text { Klingebiel \& Montgomery }\end{array} \\
\text { I }\end{array}$} \\
\hline A & $\begin{array}{l}\text { Pocas o ninguna limitación. Sin riesgo de ero- } \\
\text { sión o con riesgos ligeros. Susceptible de utiliza- } \\
\text { ción agrícola intensival. }\end{array}$ & \\
\hline B & $\begin{array}{l}\text { Moderadas limitaciones. Riesgo de erosión mo- } \\
\text { derado. Susceptible de utilización agrícola mode- } \\
\text { radamente intensiva. }\end{array}$ & II \\
\hline $\mathrm{C}$ & $\begin{array}{l}\text { Limitaciones acentuadas. Riesgo de erosión ele- } \\
\text { vado. Susceptible de utilización agrícola poco in- } \\
\text { tensiva. }\end{array}$ & III y IV \\
\hline $\mathrm{D}$ & $\begin{array}{l}\text { Limitaciones severas. Riesgo de erosión elevado } \\
\text { a muy elevado. No es susceptible de utilización } \\
\text { agrícola, salvo casos muy especiales. Pocas o } \\
\text { moderadas limitaciones para pastos, explotación } \\
\text { de monte bajo o explotación forestal. }\end{array}$ & V y VI \\
\hline $\mathrm{E}$ & $\begin{array}{l}\text { Limitaciones muy severas. Riesgo de erosión } \\
\text { muy elevado. No es susceptible de utilización } \\
\text { agrícola. Severas a muy severas limitaciones } \\
\text { para pastos, explotación de monte bajo y explo- } \\
\text { tación forestal. En muchos casos el suelo no es } \\
\text { susceptible de ninguna utilización económica: } \\
\text { puede destinarse a vegetación natural, bosque } \\
\text { de protección o de recuperación. }\end{array}$ & VII y VIII \\
\hline
\end{tabular}

1990), asume íntegramente los principios de evaluación desarrollados en Valencia. La misma característica se observa en las aportaciones desarrolladas por Matarredona (1988) o De la Horra (1993).

Las diferencias más importantes entre las distintas aplicaciones metodológicas de carácter categórico realizadas en España pueden resumirse en los siguientes aspectos: varía la combinación de factores en la definición de las clases de Capacidad de Uso; no son idénti- 
cos al número y tipo de los factores limitantes seleccionados; existen importantes variaciones en el orden de prelación otorgado a cada factor, y los rangos de los caracteres que intervienen en la evaluación y limitan los usos del territorio se adaptan a circunstancias ambientales concretas.

Inconvenientes de la Clasificación de Capacidades Agrológicas.-La clasificación de Capacidades Agrológicas, origen de todos los sistemas de evaluación categóricos elaborados en España, es la metodología que ha recibido un mayor número de críticas, algunas justificadas, aunque otras son la consecuencia de intentar alcanzar unos resultados imposibles de obtener aplicando este método (v.gr. esta metodología no está diseñada para evaluar la capacidad de un suelo para acoger cultivos específicos). Entre los aspectos más criticados y los principales inconvenientes derivados de su aplicación destacamos los siguientes:

1. En la definición de los diferentes niveles de clasificación se adoptan unos planteamientos que sólo son válidos para las condiciones de Estados Unidos. Así, el sistema excluye las consideracines de carácter social o económico, asumiendo, implícitamente, un nivel tecnológico elevado y un uso adecuado del medio propios de los Estados Unidos.

2. Es difícil repercutir en la evaluación las modificaciones provocadas por la eliminación de los factores limitantes.

3. Las utilizaciones para las que se clasifica el suelo se definen en términos muy generales (Zonneveld, 1984). Además, no se precisan los criterios utilizados para diferenciar las distintas categorías (Breimer et al., 1986).

4. En áreas física y económicamente marginales no aporta la información necesaria para realizar una planificación de usos del suelo que permita establecer un desarrollo rural integral (Purnell, 1986). Por regla general, los resultados obtenidos de la evaluación de la tierra para la utilización forestal o para el pastoreo son inadecuados o insatisfactorios. Además, es complicada la comparación objetiva de diferentes alternativas de utilización del suelo par una misma porción territorial (Zonneveld, 1984; Purnell, 1986).

5. El orden de clasificación jerárquico, a pesar del aspecto positivo que hemos indicado con anterioridad, puede ofrecer una impresión errónea sobre el potencial real del territorio. En muchas ocasio- 
ANALISIS Y VALORACIÓN DE LOS SISTEMAS DE EVALUACIÓN DE SUELOS...

nes no permite diferenciar, en el mismo área de estudio, entre los mejores suelos agrícolas, carentes de limitaciones, de aquellos otros con algún rasgo limitante muy acusado pero muy aptos para acoger determinados usos agrícolas. Estos últimos suelos, con una gestión adecuada, son, algunas veces, más rentables que los caracterizados con la clase más alta de capacidad (Dent y Young, 1981).

6. No se presentan los valores de todos los factores limitantes que pueden afectar a la capacidad de uso de un suelo, restringiendo, por tanto, el número de limitaciones con la consiguiente pérdida de información para el usuario (Sánchez y Añó, 1993).

7. La aplicación de este sistema cualitiativo de evaluación pueden ser subjetiva, dependiendo los resultados finales de la experiencia previa del evaluador sobre los suelos a evaluar (Porta et al., 1994). De este modo, en el análisis de un mismo espacio puede surgir conclusiones diferentes (Davidson, 1985). Sin embargo, la subjetividad es evidente, sin enmascarar los resultados mediante fórmulas matemáticas, formalmente objetivas, aunque la subjetividad previa del evaluador sea muy elevada.

\section{Sistemas paramétricos de evaluación}

Los sistemas paramétricos de evaluación, en un intento de superar los planteamientos cualitativos de los sistemas categóricos, establecen, por medio de modelos matemáticos, valoraciones cuantitativas, teóricamente imparciales, para evaluar la capacidad productiva de los suelos.

Desde la década de 1920 se han desarrollado diversas metodologías de carácter paramétrico que, paulatinamente, han incorporado un mayor número de factores para evaluar la productividad de los suelos. Mientras unos sistemas son muy poco conocidos, restringidos al ámbito local o regional, otros son aceptados internacionalmente, probablemente por su incorporación a la legislación tributaria. Entre estos últimos métodos de evaluación de suelos destaca el índice de Storie (1970), sistema multiplicativo de carácter inductivo (Huddleston, 1984) que considera al suelo como un factor determinante en la productividad de los cultivos agrícolas. El índice de Storie es un modelo caracterizado por su flexibilidad, permitiendo la introducción de 
nuevos factores, v.gr. el clima, en función de las peculiaridades ambientales del área de estudio. Esta característica ha provado la aparición de múltiples adaptaciones o ampliaciones del sistema original. Es España los intentos de aplicación de este método han sido muy escasos, obteniendo unos resultados muy poco representativos.

Un mayor éxito ha tenido la difusión y aceptación del sistema de Riquier, Bramao y Cornet (1970). Estos autores establecen para tres usos (pastos, cultivos agrícolas y plantas de enraizamiento profundo) un «índice de productividad» $\mathrm{y}$ un «índice de potencialidad» o de productividad potencial. El primer índice refleja numéricamente la capacidad actual de un suelo para conseguir un rendimiento de un determinado cultivo, independientemente de los factores económicos que puedan condicionar el desarrollo de determinadas orientaciones productivas. El segundo índice expresa, también numéricamente, la productividad potencial del suelo cuando se han realizado todas las enmiendas necesarias, previamente determinadas e incluyendo las más costosas, que permitan superar los factores que condicionaban negativamente la productividad del suelo.

Por tanto, la premisa fundamental de esta metodología se ampara en considerar que la productividad agraria, con unas prácticas óptimas de manejo y unas condiciones externas al medio edáfico favorables (v.gr. carencia de enfermedades o utilización de variedades de plantas adaptadas a las condiciones climáticas), estará en función de las características intrínsecas de los suelos, agrupadas en una serie de factores que permitirán calcular el índice de productividad y de potencialidad (Ip): humedad del suelo (H), drenaje (D), profundidad efectiva (P), textura/estructura (T), saturación de bases en el complejo absorbente $(\mathrm{N})$, concentración de sales solubles (S), contenido en materia orgánica $(\mathrm{O})$, capacidad de intercambio catiónico/naturaleza de la arcilla (A) y reservas minerales (M): Ip: $\mathrm{HxDxPxTx}(\mathrm{N}$ ó S)xO$\mathrm{xAxM}$

A cada factor se asigna un valor numérico, entre 0 y 100 , en función de su incidencia sobre el rendimiento de los cultivos; posteriormente se combinan estos valores, generando un índice final que es el producto de todos los parámetros, también situado en una escala de 0 a 100. En función del valor obtenido en el índice el suelo se clasifica en cinco clases diferentes de productividad (Cuadro III).

Ventajas del sistema Riquier-Bramao-Cornet.-Este sistema de eva- 
ANÁLISIS Y VALORACIÓN DE LOS SISTEMAS DE EVALUACIÓN DE SUELOS...

CuAdro III

CLASES DE PRODUCTIVIDAD (P) Y DE POTENCIALIDAD (P') (SISTMA RIQUIER, BRAMAO Y CORNET, 1970)

\begin{tabular}{c|l|c|c|l}
\hline P & \multicolumn{1}{|c|}{ Clase } & Valor índice & P' & Interpretación para cultivos \\
\hline 1 & Excelente & $65-100$ & I & $\begin{array}{l}\text { Suelos adecuados para todos los } \\
\text { cultivos agrícolas }\end{array}$ \\
\hline 2 & Buena & $35-64$ & II & $\begin{array}{l}\text { Suelos adecuados para todos los } \\
\text { cultivos agrícolas }\end{array}$ \\
\hline 3 & Media & $20-34$ & III & $\begin{array}{l}\text { Suelos marginales para cultivos ár- } \\
\text { boles no forestales }\end{array}$ \\
\hline 4 & Pobre & $8-19$ & IV & $\begin{array}{l}\text { Suelos adecuados para pastoreo, } \\
\text { repoblación forestal, recreo o culti- } \\
\text { vos especiales (arroz) }\end{array}$ \\
\hline 5 & Muy pobre o nula & $0-7$ & V & $\begin{array}{l}\text { Suelos no adecuados para el culti- } \\
\text { vo }\end{array}$ \\
\hline
\end{tabular}

luación, igual que la metodología de capacidades agrológicas, es flexible: la clasificación puede adaptarse a condiciones ambientales diferentes y a distintas escalas de trabajo. Además, su utilización presenta una serie de ventajas respecto a los sistemas categóricos:

1. Carácter cuantitativo, preciso y, aparentemente, objetivo. De este modo atenúa, teóricamente, la subjetividad del evaluador.

2. El análisis de cada uno de los factores, reflejado en valores individuales, permite determinar los parámetros que condicionan un nivel concreto de productividad.

3. Valora las interacciones entre todos los factores que se han seleccionado para deterinar la prouctividad, estableciendo una escala continua de clasificación del suelo.

4. Está diseñado para repercutir en la evaluación la superación o eliminación de los factores limitantes, que, en última instancia, determinará un nivel diferente de productividad.

5. Las conclusiones de la evaluación, plasmadas en cartografías temáticas, reflejan tanto la productividad actual como la potencial. 
Aplicación en España. Caracteristicas.-En España, respecto al método original, se ha propuesto un nuevo parámetro, la caliza, en su doble aspecto, total y activa, factor importante para establecer la productividad de los cultivos de frutales en los suelos mediterráneos. Sin embargo, la aplicación de este sistema de evaluación se ha realizado, salvo escasas excepciones (De Haro, et al., 1993), sin apenas modificaciones respecto al método original, independientemente del ámbito geográfico de aplicación: Camp de Tarragona (Porta, et al., 1980), Rioja Alavesa (Ocio, et al., 1987) o el valle del Iregua (Andrades, et al., 1993). A nivel del área mediterránea, entre las aportaciones metodológicas más recientes, destacan el índice de fertilidad diseñado por Herrero-Borgoñón y Rubio (1994) para su aplicación en zonas forestales de la provincia de Valencia, y la propuesta, en combinación con los niveles jerárquicos establecidos por la F.A.O. (1976), de Aguilar y Ortiz (1992), aplicada en sectores de Murcia.

Las principales diferencias entre los distintos planteamientos metodológicos de tipo paramétrico radican en los atributos del medio físico considerados, la importancia otorgada a cada propiedad del suelo y en el tipo de fórmula matemática utilizada, opciones que se reflejarán en la estimación cuantitativa final.

Inconvenientes de los sistemas paramétricos.-La utilización de esta última metodología, en general todos los sistemas paramétricos de evaluación de suelos, también presenta inconvenientes, destacando los siguientes:

1. El sistema no considera todos los parámetros que condicionan la capacidad productiva de un suelo. Actualmente todavía no se ha diseñado un modelo matemático capaz de mostrar, objetivamente, las interacciones entre todos los factores que determinan la productivad del sistema edáfico.

2. El método simplifica la influencia de las características climáticas. Además, no valora las condiciones de uso o manejo de la tierra (Boixadera y Porta, 1991).

3. La puntuación parcial de los parámetros es muy arbitraria, provocando, en muchas ocasiones, que el valor asignado a algunos factores sea excesivo -o muy alto o muy bajo-. Por el contrario, no intervienen o se omiten deliberadamente otros parámetros que pueden ser más determinantes para evaluar adecuadamente la productividad. 
4. Los resultados finales, a pesar de su exactitud, muchas veces no refleja adecuadamente la realidad agraria del área de estudio. En función de su carácter multiplicativo, la incidencia tan determinante que posee en el sistema la propiedad edáfica con una puntuación más desfavorable, provoca que la presencia de un único factor limitante muy relevante sea motivo suficiente para que disminuyan considerablemente los valores establecidos en el índice final. Valores que no concuerdan con la realidad agraria de determinados espacios rurales, donde, en muchas ocasiones, es posible acceder a cosechas económicamente aceptables aplicando la tecnología adecuada o cultivando especies menos exigentes (Boixadera y Porta, 1991).

5. Los sistemas paramétricos, en última instancia, no son más que expresiones matemáticas de conceptos subjetivos, sólo es objetivo el tratamiento matemático de los factores (McRae y Burnham, 1981). Por tanto, la objetividad y precisión es aparente ya que existe un alto grado de subjetividad en la selección de las variables que intervienen en la evaluación, en la interacción de los distintos factores y en la manera con que las combinaciones de los parámetros determinan la productividad del suelo.

Esquema F.A.O. de Evaluación de Tierras. Caracteristicas.

A diferencia de los sistemas de evaluación de propósitos generales, ya sean categóricos o paramétricos, la Organización de las Naciones Unidas para la Agricultura y la Alimentación (F.A.O.) aporta un conjunto de principio, conceptos y procedimiento metodológicos que permiten evaluar la aptitud de la tierra en función de tipos específicos de uso del suelo, definidos en términos muy precisos.

El esquema de evaluación de tierras de la F.A.O. (1976) se concibió inicialmente como un soporte metodológico para la planificación de los usos agrarios del territorio, sobre todo en los países subdesarrollados. Esta nueva propuesta surge en un momento (década de 1970) marcado por la necesidad de utilizar adecuadamente el suelo, sometido, en amplias zonas de naciones económicamente subdesarrolladas, a intensos procesos de degradación provocados por la presión ejercida por el fuerte crecimiento demográfico y el desarrollo anárquico de las actividades productivas, que impedían al sector agrario 
abastecer adecuadamente de alimentos a la población. Por tanto, en este ámbito las aptitudes del suelo casi siempre se expresarán en incrementos cuantitativos de la producción relacionados con unas condiciones biofísicas más o menos favorables. Posteriormente los procedimientos se adaptaron a las condiciones de los países europeos, siendo aceptados como un instrumento metodológico básico para la planificación de usos del suelo en los países que integran la U.E. (Verheye, 1986 y 1991).

La estructura de clasificación en niveles jerárquicos de aptitud -órdenes, clases, subclases y unidades- (Cuadro IV) que establece la F.A.O. (1976) es muy parecida a la clasificación de capacidades agrológicas, e, igual que las metodologías anteriores, es un sistema de evaluación muy flexible, permitiendo al evaluador seleccionar, entre todos los procedimientos metodológicos que ofrecen las diferentes guías de la F.A.O., aquéllos más apropiados a las condiciones físicas,

Cuadro IV

ESTRUCTURA ESQUEMÁTICA DE LA CLASIFICACIÓN DE APTITUD DE LA TIERRA (SISTEMA F.A.O.)

\begin{tabular}{c|c|c|c}
\hline \multirow{2}{*}{ Orden } & Clase & Subclase & Unidad \\
\hline \multirow{3}{*}{ S- Apta } & S1 & --- & -- \\
\cline { 2 - 4 } & S2 & S2d S2e S2de, etc. & S2e(1) S2e(2), etc. \\
\cline { 2 - 4 } & S3 & & \\
\hline \multirow{2}{*}{ N- No Apta } & N1 & N1d N1e, etc. & $-\ldots$ \\
\cline { 2 - 4 } & N2 & $-\ldots-\ldots$ & $-\ldots$ \\
\hline
\end{tabular}

Orden. Reflejan el tipo de aptitud: Apto (S) - No Apto (N).

Clase. Reflejan el grado de aptitud de cada orden: Muy Apto (S1) - Moderadamente Apto (S2) - Marginalmente Apto (S3) - No Apto Actualmente (N1) - No Apto Permanentemente (N2).

Subclase. Expresan las limitaciones de las tierras: v.gr. $w$ (deficiencias de drenaje), $e$ (erosión), etc.

Unidad. Indica diferencias de escasa importancia en las características de producción o en los requisitos de gestión. 
económicas y sociales de la zona objeto de la evaluación (Young, 1985; Purnell, 1984), independientemente del tipo de escala a emplear o de las clases de utilizaciones alternativas del territorio a estudiar. Sin embargo el sistema de la F.A.O. presenta, en algunos aspectos, unas características distintas que pueden resumirse en varios puntos:

1. La F.A.O. aporta un sistema abierto de evaluación, así su utilización obliga a desarrollarlo para cada aplicación concreta.

2. Los procedimientos de evaluación se adaptan a las necesidades de la planificación de usos del suelo (Dent y Young, 1981; Zonneveld, 1984; Purnell, 1986), sobre todo al incorporar un nuevo concepto («land»-tierra-) más amplio y menos limitado que el concepto suelo.

3. La aptitud de la tierra se valora en función de tipos específicos de utilización, partiendo de un principio básico: cuando las utilizaciones del suelo son diferentes, las condiciones de uso también son distintas.

4. La evaluación siempre tiene que comparar varios tipos de utilización y asignar a cada uno de ellos un grado determinado de aptitud. La propuesta final de uso del suelo ha de evitar cualquier manifestación de degradación medioambiental, sin establecer, a diferencia de la clasificación de capacidades agrológicas, una gradación jerárquica en la tipología de utilizaciones de las tierras.

5. Los procedimientos de la F.A.O. introducen en el proceso evaluador el análisis social, económico y financiero de las propuestas de utilización del suelo. Esta valoración se realiza a continuación de la clasificación cualitativa del territorio, constituyendo en terminología F.A.O. la solución bifásica, o de forma simultánea a la evaluación de los factores físicos de las tierras, opción recomendada por la F.A.O. Por tanto, además de la idoneidad física de un uso se indica su viabilidad económica y financiera a largo plazo. De este modo, tienen que integrarse en el proceso de evaluación las aportaciones de disciplinas muy diversas, planteando, por tanto, una evaluación de carácter multidisciplinar.

Problemas en la aplicación del sistema F.A.O.-La labor iniciada por la F.A.O. en 1976, ha continuado con la difusión de una serie de directivas que han desarrollado los conceptos básicos y los procedimientos de evaluación iniciales, circunscribiendo el ámbi- 
to de aplicación de la evaluación a los principales tipos de uso de la tierra (F.A.O. 1983, 1984 y 1990). Actualmente, sus procedimientos de evaluación son muy utilizados para evaluar los suelos en los estudios de planificación de usos del territorio, aunque existen aspectos criticables, entre los cuales deben destacarse los siguientes:

1. Existe una confusión conceptual muy acusada, consecuencia de la redefinición terminológica de conceptos preexistentes que generan múltiples interpretaciones (Van Diepen, et al., 1991; Meliz, 1986). Además, algunos principios fundamentales del sistema o incluso los procedimientos de evaluación están definidos con muy poca precisión.

2. La conexión entre los procedimientos de evaluación y la planificación de usos del territorio está muy poco articulada, sin integrar adecuadamente la información procedente de otras disciplinas. Además, las directivas de la F.A.O., en gran medida, continúan patrocinando procedimientos de evaluación basados en el conocimiento intuitivo del experto (Van Diepen, et al., 1991).

3. En algunas ocasiones la tolerancia de ciertos cultivos a limitaciones específicas del medio se conocen con muy poca precisión. La utilización de información deficiente o la ausencia de datos contrastados en la definición de las condiciones de uso, pueden generar conclusiones imprudentes cuando no se demora la planificación hasta completar investigaciones que validen la actuación de las utilizaciones del medio recomendadas prematuramente.

4. Actualmente, y a pesar de las recomendaciones de la F.A.O., predomina la evaluación cualitativa sobre la cuantitativa, sin incorporar, en muchas ocasiones, el análisis financiero o económico de los usos propuestos o aportando una valoración muy general. En estos momentos todavía no se han resuelto las dificultades provocadas por las fluctuaciones temporales de los precios de los productos agrarios o de los costes de producción. También suele ser muy siemple o muy general el análisis de la estructura socioeconómica.

5. En los países subdesarrollados la aplicación de este sistema de evaluación se ha realizado sin valorar los conflictos sociales o la inestabilidad política. En ningún caso se plantea que, probablemente, una distribución más equitativa y una tenencia más segura de la propiedad de la tierra podría ser el instrumento fundamental para utili- 
ANÁLISIS Y VALORACIÓN DE LOS SISTEMAS DE EVALUACIÓN DE SUELOS...

zar el suelo en su uso más apto, mejorando tanto la producción como la conservación de recurso edáfico.

Aplicación en España. Peculiaridades.-En España también se han desarrollado metodologías que utilizan los principios y conceptos básicos del sistema F.A.O. Las propuestas más interesantes son las que se han diseñado para su aplicación en Andalucía (De la Rosa y Moreira, 1987; Sánchez Marañón, 1990; Moreira, 1991; De la Rosa, 1996), Galicia (Díaz-Fierros y Gil, 1984) y, sobre todo, el procedimiento de evaluación de tierras con una finalidad fiscal aplicado inicialmente en El Prat de Llobregat (Boixadera y Porta, 1991). Esta última metodología, a pesar de las carencias detectadas (v.gr., carece de información agronómica sobre los rendimientos productivos de los cultivos para condiciones concretas de manejo; no considera la introducción de mejoras en el suelo o las posibles modificaciones de su uso; el método sólo se aplica en un ámbito geográfico muy reducido dedicado al cultivo intensivo de regadío, etc.) constituye, actualmente, la aportación que mejor ha desarrollado en España los criterios de evaluación diseñados por la F.A.O.

En conjunto, la utilización del sistema F.A.O. en nuestro país presenta dos características:

1. La flexibilidad que caracteriza a este sistema de evaluación ha provocado que se empleen únicamente los procedimientos más simples. Además, la mayoría de las metodologías utilizan de manera muy poco estricta los principios y los conceptos básicos del sistema o, incluso, la nomenclatura que muchas veces enmascara el uso de criterios de evaluación, sobre todo, categóricos.

2. Predomina un tipo de evaluación que siempre opta por la solución bifásica, por tanto sólo incorpora la evaluación de los factores físicos de las tierras, obviando, directamente, las consideraciones de carácter social, económico y financiero, o aplazando su análisis a un futuro, cuando menos, incierto.

\section{El Plan Nacional de Cartografía Temática Ambiental}

Los mapas de suelos siempre han supuesto, en la mayoría de las ocasiones, el soporte de la evaluación, sin embargo, en las últimas dos décadas, se ha cuestionado la utilidad de los estudios de suelos

$$
-347-
$$


(Ibáñez, et al., 1993). Más aún, con demasiada frecuencia la información edafológica se infrautiliza o incluso se desestima. Entre las principales causas que provocan esta situación destacan las siguientes: presentación inadecuada de los resultados; utilización de una terminología especializada, poco accesible a científicos procedentes de otras disciplinas; ausencia de un sistema general de clasificación aceptado por toda la comunidad científica; pérdida de información producida durante el proceso de elaboración de la cartografía, etc. (Dudal, 1986, en Ibáñez, et al., 1993).

En este contexto hay que inscribir la propuesta de la cartografía de suelos (Sánchez, et al., 1996) del Plan Nacional de Cartografía Temática Ambiental (P.N.C.T.A.) que pretende obtener mapas que aporten información útil para la evaluación y planificación del territorio. Con este objetivo se han incorporado a la cartografía dos elementos novedosos: el Indicador de Capacidad y el Indicador de Vulnerabilidad (Añó, 1996). Así, las unidades cartográficas se establecerán en base a los tipos de suelos (considerando tanto los suelos dominante como las inclusiones) y a los dos Indicadores. De esta forma, en el mapa de suelos, una unidad cartográfica tendrá el mismo significado en relación a los siguientes elementos:

Tipología del suelo: utilizando el sistema de clasificación Soil Taxonomy (Soil Survey Staff, 1994) y su correlación con el sistema FAO (FAO-UNESCO, 1988).

Indicador de capacidad: refleja la actuación de un conjunto de parámetros del suelo y del entorno físico: disponibilidad de agua para las plantas, condiciones térmicas, pendiente, riesgo de inundación, espesor efectivo, afloramientos rocosos, pedregosidad superficial o en la zona radicular, hidromorfia, clase textural, propiedades químicas, alcalinidad, salinidad y grado de erosión hídrica actual. Así, este Indicador muestra la vocación intrínseca del medio edáfico que permitirá o limitará el uso agrario.

Indicador de vulnerabilidad: refleja las limitaciones (erosión potencial, riesgo de salinización o alcalinización y susceptibilidad del suelo a la contaminación) que puede restringir las posibilidades de uso del suelo o repercutir negativamente en su potencial de utilización. En última instancia este Indicador estima la facilidad con que las actividades antrópicas, por prácticas inadecuadas o la ausencia de medidas efectivas de control, pueden afectar las funciones ecológicas o 
agronómicas que un determinado tipo de suelo puede llegar a desarrollar.

Por tanto, la unidad cartográfica del mapa de suelos del P.N.C.T.A. es una porción del territorio, identificada y delimitada a base de aspectos externos (relieve, formas de la pendiente, condiciones de drenaje, etc.), que se caracteriza por poseer una serie de características intrínsecas y extrínsecas del suelo semejantes, de tal manera que sea posible en toda su demarcación un mismo uso continuado del suelo, una idéntica evaluación, los riesgos naturales se presentan con igual intensidad y responde de la misma manera ante una perturbación, actuación o impacto que incida sobre ella.

\section{Conclusiones}

La revisión metodológica que hemos realizado pone de manifiesto que la Evaluación de Suelos en España se caracteriza por aplicar, sin incorporar ningún tipo de modificación, los criterios de evaluación de los sistemas más aceptados internacionalmente, o por emplear variantes de estos métodos adaptados a condiciones ambientales comarcales o regionales. En la década de los setenta y parte de los ochenta ha predominado la utilización de los sistemas de evaluación de propósitos generales (categóricos o paramétricos). Por el contrario, desde finales de la década anterior el panorama evaluador está dominado por el sistema de la F.A.O., tendencia que continúa actualmente a pesar de la confusión conceptual, la utilización imprecisa o incorrecta de los criterios de evaluación, o la escasa conexión entre los procedimientos metodológicos propuestos y la planificación de usos del suelo.

La aplicación en España de las normas metodológicas de los principales sistemas internacionales de evaluación de suelos no aporta resultados satisfactorios. Estos métodos se han ideado para resolver una problemática determinada: unos más interesados en el incremento cuantitativo de la producción, otros más proteccionistas del medio, pero, en conjunto, ninguno valora adecuadamente las características y problemas específicos de nuestro territorio. Las modificaciones o redefiniciones locales en muy pocas ocasiones 
pueden soslayar las carencias e inconvenientes que se han analizado, sobre todo cuando se utilizan criterios originalmente mal seleccionados o se emplean los mismos límites cuantificados que permiten distinguir los rangos de los factores. Por tanto, consideramos que para juzgar la capacidad o aptitud de los suelos presentes en un territorio es necesario desarrollar y aplicar un sistema de evaluación adaptado a sus peculiaridades, necesidades y problemas, y que sea capaz de generar un tipo de información útil para la planificación territorial.

Con este propósito, la cartografía de suelos del Plan Nacional de Cartografía Temática Ambiental ha incorporado dos elementos innovadores para evaluar la potencialidad y fragilidad del recurso edáfico: el Indicador de Capacidad y de Vulnerabilidad, diseñados en función de las características medioambientales mediterráneas.

\section{BIBLIOGRAFÍA}

Aguilar, J. y Oortiz, R. (1992): «Metodología de capacidad de uso agrícola de suelos», III Congreso Nacional de la Ciencia del Suelo, pp. 281-286.

Andrades, M.; Ballesta, R. J.; Carral, P. y Martínez, E. (1993): «Evaluación paramétrica de los suelos del valle del Iregua (La Rioja)», V R.N. Geología Ambiental y Ordenación del Territorio, pp. 543-552.

AÑó, C. (1996): Metodología de evaluación de suelos para el ámbito mediterráneo, tesis doctoral, Facultad de Geografía e Historia. Universitar de València. 200 pp.

Azevedo, A. L. y Cardoso, J. C. (1962): «Soil classification in Portugal and its application in agricultural research», Trans. Comm. IV y V. International Society of Soil Science, pp. 473-479.

Badía, D.; Martí, C. y Alcañiz, J. M. (1993): «Evaluación de la capacidad agrológica de la comarca de la Alta Garrotxa (Girona)», V R. N. Geología Ambiental y Ordenación del Territorio, pp. 509-516.

BEEK, J. K. (1981): «From soil survey interpretation to land evaluation. Part 2. From the present to the future», Soil Survey and Land Evaluation, vol. n. ${ }^{\circ}$ 2, pp. 18-25.

Boixadera, J. y Porta, J. (1991): Información de suelos y evaluación catastral. Método del Valor Índice. Madrid, Ministerio de Economía y Hacienda, 151 pp.

Breimer, R. F.; Van KeKem, A. J. y Van Reuler, H. (1986): Guidelines for soil survey and land evaluation in ecological research, MAB Technical Notes, 17, París, UNESCO, $124 \mathrm{pp}$.

C.O.P.U.T. (1991): Sistema de Información Territorial, València, Conselleria d'Obres Públiques, Urbanisme i Transports. Generalitat Valenciana, 49 pp.

Davidson, D. A. (Ed.) (1985): Land Evaluation, Nueva York, Van Nostrand Reinhold Soil Science Series, $373 \mathrm{pp}$.

- (1992): The evaluation of Land Resources, Londres, Longman Scientific \& Technical, $198 \mathrm{pp}$.

De Haro, S.; Agullar, J.; Fernandez, J. y Fernández, E. (1993): «Evaluación de suelos en la comarca olivarera de Martos (Jaen)», VR. N. Geologia Ambiental y Ordenación del Territorio, pp. 575-583. 
DE la Horra, J. L. (1993): «Cartografía edáfica y de capacidad de uso en ambientes semiáridos. La comarca de Torrijos (Toledo)», XII Congreso Latinoamericano de la Ciencia del Suelo, pp. 1302-1310.

DE LA Rosa, D. (Ed.) (1996): MicroLEIS 4.1 P.I.: Sistema Informático para la Transferencia de Datos y Evaluación Agro-ecológica de Tierras. Software y documentación, Sevilla, pub. IRNAS, CSIC, $433 \mathrm{pp}$.

- y Moreira, J. M. (1987): Evaluación ecológica de recursos naturales de Andalucía, Sevilla, Agencia de Medio Ambiente, Junta de Andalucía, 130 pp.

Delgado, G. y Agullar, J. (1985): «Caracterización de la capacidad agrológica de los suelos de la zona de Linares basada en un mapa edafológico», Anal. Edaf. Agrobiol., t. XLIII, n. ${ }^{\circ} 11-12$, pp. 1515-1525.

Dent, D. y Young, A. (1981): Soil survey and land evaluation, Londres, Georges Allen \& Unwin, $278 \mathrm{pp}$.

Díaz-Fierros, F. y Gil, F. (1984): Capacidad productiva de los suelos de Galicia, Santiago de Compostela, Universidad de Santiago, $82 \mathrm{pp}$.

Dumanski, J.; Marshall, I. B. y Huffman, E. C. (1979): «Soil capability analysis for regional land use planning. A study of the Ottawa urban fringe», Canadian Journal of Soil Science, n. $^{\circ} 59$, pp. $363-379$.

F.A.O. (1976): Esquema para la evaluación de tierras, Roma, Boletín de Suelos, n. ${ }^{\circ} 32$. F.A.O., 66 pp.

- (1983): Guidelines: land evaluation for rainfed agriculture, Roma, Soils Bulletin, n. ${ }^{\circ} 52$. F.A.O., 237 pp.

- (1984): Land evaluation for forestry, Roma, Forestruy Paper, n. ${ }^{\circ} 48$. F.A.O., 123 pp.

- (1990): Evaluación de tierras para la agricultura en regadio: directivas, Roma, Boletín de Suelos, n. ${ }^{\circ}$ 55. F.A.O., 289 pp.

F.A.O.-U.N.E.S.C.O. (1988): Soil Map of the World. Revised Legend, Roma, World Soil Resources Report 60. F.A.O., 119 pp.

HERRERO-Borgoñon, J. J. y J. L. (1994): Impacto de las técnicas forestales de repoblación sobre los procesos erosivos y la fertilidad del suelo en condiciones ambientales mediterráneas, València, Consellería d'Agricultura, Pesca i Alimentació. Generalitat Valenciana, $256 \mathrm{pp}$.

HudDLESTON, J. H. (1984): «Development and use of soil productivity ratings in the U.S.», Geoderma, 32, pp. 297-317.

IBAÑEZ, J. J.; ZINCK, A. J. y JimÉNEZ, R. (1993): «Soil survey: old and new challenges», I.T.C. Journal 1, pp. 7-13.

KLINGEBIEL, A. A. y MonTgomery, P. H. (1961): Land capability classification, Agricultural Handbook 210. Washington D.C., Soil Conservation Service. U.S.D.A., 21 pp.

M.A.P.A. (1974): Caracterización de la capacidad agrológica de los suelos de España. Metodología y normas, Madrid, D. G. de Producción Agraria. Ministerio de Agricultura, Pesca y Alimentación.

Matarredona, E. (1988): «Capacidad de uso del suelos del País Valenciano», Estudios Geográficos, t. XLIX, n. ${ }^{\circ} 190$, pp. 99-107.

McRae, S. G. y Burnham, C. P. (1981): Land evaluation, Oxford, Clarendon Press, 239 pp.

Melitz, P. J. (1986): «The sufficiency concept in land evaluation», Soil Survey and Land Evaluation, vol. 6, n. ${ }^{\circ}$ 1, pp. 9-19.

Monturiol, F. y AlCAlA, L. (1990): Mapa de capacidad potencial de uso agrícola de la Co munidad de Madrid, Madrid, Consejería de Agricultura y Cooperación-C.S.I.C., 31 pp.

MoReIRA, J. M. (1991): Capacidad de uso y erosión de suelos. Una aproximación a la evaluación de tierras en Andalucía, Sevilla, A.M.A. Junta de Andalucía, 446 pp.

OcIo, J. A.; JIMÉNEZ, R. y GuERRA, A. (1987): «Aproximación a la evaluación paramétrica de suelos, para distintos usos, en Rioja Alavesa», III R. N. Geología Ambiental y Ordenación del Territorio, pp. 1333-1350.

$$
-351-
$$


OLson, G. W. (1984): Field guide to soils and the environment. Applications of soil surveys, Nueva York, Chapman and Hall, 178 pp.

Porta, J.; López-Acevedo, M. y Marqués, M. A. (1980): «Aportación del sistema RiquierFAO a la ordenación territorial: aplicación a dos áreas modelo del campo de Tarragona», I R. N. Geología Ambiental y Ordenación del Territorio, 14 pp.

-; - y Roquero, C. (1994): «Usos y aplicaciones de los mapas de suelos», en Edafología para la agricultura y el medio ambiente, Madrid, Mundi Prensa, pp. 551-587.

Purnell, M. F. (1984): "The FAO methods of land evaluation for agriculture and forestry as compared to extensive grazing», en W. Siderius (ed.), Proceedings of the workshop on land evaluation for extensive grazing (LEEG), Wageningen, pub. 36, I.L.R.I., pp. 133-143.

- (1986): "Aplication of thr FAO framework for land evaluation for conservation and land use planning in sloping areas: potentials and constraits", en W. Siderius (ed.), Land evaluation for land-use planning and conservation in sloping areas, Wageningen, pub. 40, I.L.R.I., pp. 17-31.

Riquier, J.; Bramao, L. y Cornet, S. P. (1970): A new system or soil appraisal in terms of actual and potential productivity, Roma, FAO Soil Resources, Development and Conservation Service, Land and Water Development División, F.A.O., 38 p.

SÁnchez, J. y AÑó, C. (1993): «Metodología de capacidad de uso para la planificación de usos del suelo en el ámbito mediterráneo», XII Congreso Latinoamericano de la Ciencia del Suelo, pp. 1391-1398.

-; Colomer, J. C. y Nieves, M. (1996): Norma técnica para la elaboración del P.N.C.T.A. Cartografia de suelos escala 1:50.000, 26 pp. Inédito.

-; Rubio, J. L.; Martínez, V. y Antolín, C. (1984): «Metodología de capacidad de uso de los suelos para la cuenca mediterránea», I Congreso Nacional de la Ciencia del Suelo, pp. 937-948.

SÁnchez MARAÑón, M. (1990): Cartografía y evaluación de los suelos en las sierras subbéticas de la provincia de Córdoba, Sevilla, Instituto Andaluz de Reforma Agraria, 219 $\mathrm{pp}$.

Soil Survey Staff (1994): Keys to soil taxonomy, Blacksburg, Soil Conservation Service. U.S.D.A., 524 pp.

Storie, R. E. (1970): Manual de evaluación de suelos, México, UTEHA, 225 pp.

Van Diepen, C. A.; Van Keulen, H.; Wolf, J. y Berkout, J. (1991): «Land evaluation: from intuition to quantification», Advances in Soil Science, vol. 15, pp. 139-204.

Verheye, W. H. (1986): «Principles of land appraisel and land use planning within the European Community», Soil Use and Management 2, pp. 120-124.

- (1991): «Aspects of land assessment for rural land use planning in industrialized countries with particular emphasis to the European Communities», Quaderni di Scienza del Suolo, vol. III, pp. 5-16.

YounG, A. (1985): «Land evaluation and agroforestry diagnosis and design: towards a reconciliation of procedures», Soil Survey and Land Evaluation, vol. 5, n. ${ }^{\circ} 3$, pp. 61 76.

ZoNNEVELD, I. S. (1984): «Principles of land evaluation for extensive grazing», en W. Siderius (ed.), Proceedings of the workshop on land evaluation for extensive grazing, Wageningen, pub. 36, I.L.R.I., pp. 84-117.

Resumen: En España la Evaluación de Suelos, dentro del contexto de la Edafología Aplicada, ha tenido un desarrollo muy tardío y, sobre todo, una evolución muy dependiente de las metodologías internacionales más significativas: la Clasificación de Capacidades Agrológicas, el sistema paramétrico de Riquier, Bramao y Cornet, y los procedimientos de evaluación establecidos por la Organización de las Naciones Unidas para la Agricultura y la Alimentación. Analizamos las principales características metodológicas y las ventajas e inconvenientes que conllevan la aplicación de estos sistemas de 
ANÁLISIS Y VALORACIÓN DE LOS SISTEMAS DE EVALUACIÓN DE SUELOS...

evaluación, cuestionando la vigencia de estas metodologías y justificamos la necesidad de establecer un marco metodológio propio, adaptado a las condiciones biofísicas de nuestro territorio. La cartografía de suelos del Plan Nacional de Cartografía Temática Ambiental refleja esta situación, incorporando el Indicador de Capacidad y de Vulnerabilidad como elementos metodológicos innovadores en la valoración del recurso suelo.

Palabras Clave: España. Cartografía de suelos. Sistemas de evaluación. Análisis metodológico.

AвsTRACT: Land Evaluation in Spain -within the context of Applied Edaphology- experienced a late development and, above all, its evolution has been highly dependent on the most significant international methodologies: the Land Capability Classification, the parametrie system of Riquier, Bramao and Cornet, and the evaluation procedures established by the Food and Agriculture Organization of the United Nations. In this paper we analyse the main methodological characteristics of these evaluation systems and the advantages and drawbacks involved in their application. We argue whether these methodologies are still valid and we point out the need to set up a proper methodological frame fitted for the biophysical traits of our country. Soil maps from the National Plan of Environmental Cartography evidence that situation and they include the Capability and Vulnerability indexes as innovator methodological elements in the assessment of the soil as a natural resource. lysis.

KeY WORDS: Spain. Soils cartography. Land evaluation systems. Methodological ana-

Résumé: En Espagne l'Evaluation des Sols, dans le contexte de l'Edaphologie Appliquée, a eu un développement très tardit et, surtout, une évolution très dépendente des méthodologies internationales les plus significatives: la Classification del Capacités Agrologiques, le système paramétrique de Riquier, Bramao et Cornet et les procédés d'évaluation établis par l'Organisation des Nations Unies pour l'Agriculture et l'Alimentation. Nous analysons les principales caractéristiques méthologiques ainsi comme les avantages et les inconvenients dérivés de leur application. Le résultat est une mise en question de la validité de ces méthodologies. C'est ainsi que nous justifions le besoin d'établir un cadre méthodologique propre adapté aux conditions biophysiques de l'Espagne. La cartographie des sols du Plan National de Cartographie de l'Environnement nous montre cette situation tout en incluant de l'Indicateur de Capacité et celui de Vulnétabilité comme des éléments méthologiques innovateurs dans la valoration la ressource sol.

Mots CLEF: Espagne. Cartographie des sols. Sistèmes d'évaluation. Analyse méthodologique. 